\title{
AN EXTENSION OF TOTAL GRAPH OVER A MODULE
}

\author{
A. ABBASI AND A. RAMIN
}

Received 01 March, 2015

\begin{abstract}
Let $R$ be a commutative ring with nonzero identity and $U(R)$ its multiplicative group of units. Let $M$ be an R-module where the collection of prime submodules is non-empty and let $N_{\Lambda}$ be an arbitrary union of prime submodules. Also, suppose that $c \in U(R)$ such that $c^{-1}=c$. We define the extended total graph of $M$ as a simple graph $T \Gamma_{c}\left(M, N_{\Lambda}\right)$ with vertex set $M$, and two distinct elements $x, y \in M$ are adjacent if and only if $x+c y \in N_{\Lambda}$. In this paper, we will study some graph theoretic results of $T \Gamma_{c}\left(M, N_{\Lambda}\right)$.
\end{abstract}

2010 Mathematics Subject Classification: 05C25; 13 C99

Keywords: total graph, prime submodule

\section{INTRODUCTION}

Let $R$ be commutative ring with $1 \neq 0, U(R)$ its multiplicative group of units and $Z(R)$ its set of zero-divisors. A proper submodule $N$ of $M$ is said to be a prime submodule if whenever $r m \in N$ for some $r \in R$ and $m \in M$, then either $m \in N$ or $r \in\left(N:_{R} M\right)$. Clearly, if $N$ is a prime submodule of $M$, then $P=$ $\left(N:_{R} M\right)$ is a prime ideal of $R$. Let $M$ be an R-module, $T(M)$ its set of torsion elements and $\left\{N_{\lambda}\right\}_{\lambda \in \Omega}$ its set of all prime submodules. The R-module $M$ is said to be primeless if $\Omega=\varnothing$. For a submodule $L$ of an R-module $M$, the ideal $\{r \in R \mid r M \subseteq$ $L\}$ and submodule $\{m \in M \mid r m \subseteq L\}$ will be denoted by $\left(L:_{R} M\right)$ and $\left(L:_{M} r\right)$, respectively. Let $N_{\Lambda}=\bigcup_{\lambda \in \Lambda} N_{\lambda}$ be a proper subset of $M$, and let $H_{\Lambda}=\left(N_{\Lambda}:_{R} M\right)$ for $\varnothing \neq \Lambda \subseteq \Omega$. It can be shown that $H_{\Lambda}=\bigcup_{\lambda \in \Lambda} P_{\lambda}$.

The total graph of $R$ was introduced by Anderson and Badawi in [4], as the graph with all elements of $R$ as vertices, and two distinct vertices $x, y \in R$ are adjacent if and only if $x+y \in Z(R)$. Also they introduced in [5] the generalized total graph of $R$ in which $Z(R)$ is extended to $H$, a multiplicative - prime subset of $R$, in such away that $a b \in H$ for every $a \in H$ and $b \in R$, and whenever $a b \in H$ for all $a, b \in R$, then either $a \in H$ or $b \in H$. In fact, it is easily seen that $H$ is a multiplicative-prime subset of $R$ if and only if $R \backslash H$ is a saturated multiplicatively closed subset of $R$. Thus $H$ is a multiplicative-prime subset of $R$ if and only if $H$ is a union of prime ideals of $R$. 
In what follows, we extend the generalized total graph such that the extended total graph of $M$ is a simple graph with vertex set $M$, and two distinct elements $x, y \in M$ are adjacent if and only if $x+c y \in N_{\Lambda}$ where $c \in U(R)$ and $c^{-1}=c$. This graph is denoted by $T \Gamma_{c}\left(M, N_{\Lambda}\right)$. In general, for $A, B \subseteq M, T \Gamma_{c}(A, B)$ is a simple graph with vertices all element of $A$, and two distinct vertices $x$ and $y$ are adjacent if and only if $x+c y \in B$.

The authors in [7] and [10] generalized the notion of a total graph to an $R$-module $M$. They considered the vertex set of a graph $T(\Gamma(M))$ as the elements of $M$ such that two vertices are adjacent if and only if $x+y \in T(M)$. In [3], D. D. Anderson and Sangmin Chun proved that if $M \neq T(M)$, then $T(M)$ is a union of prime submodules of $M$. Consequently, $T \Gamma_{c}\left(M, N_{\Lambda}\right)$ is a generalization of $T(\Gamma(M)$ too. Let $c=-1$, then $\overline{T \Gamma_{c}}\left(M, N_{\Lambda}\right)$, the complement graph of $T \Gamma_{c}\left(M, N_{\Lambda}\right)$ (i.e., $T \Gamma_{c}\left(M, M \backslash N_{\Lambda}\right)$ ), is a Cayley graph, also let $M=R$ and $N_{\Lambda}$ be the union of all the maximal ideals of $R$ (i.e., $\left.N_{\Lambda}=R \backslash U(R)\right)$, then observe that $\overline{T \Gamma_{1}}\left(M, N_{\Lambda}\right)$ (i.e., $T \Gamma_{1}\left(M, M \backslash N_{\Lambda}\right)$ ) is the unit graph of $R$ in the sense of [6] and $\overline{T \Gamma_{-1}}\left(M, N_{\Lambda}\right)$ (i.e., $T \Gamma_{-1}\left(M, M \backslash N_{\Lambda}\right)$ ) is the unitary Cayley graph in the sense of [2] and [9].

For a proper submodule $L$ of $M, M(L)=\left\{m \in M \mid r m \in L\right.$ for some $r \in R \backslash\left(L:_{R}\right.$ $M)\}$. In section 2, we will show that if $M \neq M(L)$, then there is $\Lambda \subseteq \Omega$ such that $M(L)=N_{\Lambda}$. In [1], the authors introduce a generalization of total graph of a module with respect to the set $M(L)$. For $\Lambda \subseteq \Omega, N_{\Lambda}$ is more general than $M(L)$ since there are R-modules $M$ and $\Lambda \subseteq \Omega$ such that $N_{\Lambda}$ is not of the form $M(L)$ for all submodules $L$ of $M$.

In section 3, we determine some basic properties of extended total graph specifically identifying its regularity and vertex transitivity. Since $N_{\Lambda}$ is a union of prime submodules of $M$, the study of $T \Gamma_{c}\left(M, N_{\Lambda}\right)$ breaks naturally into two cases depending on whether or not $N_{\Lambda}$ is a (prime) submodule of $M$. In section 4, we study the case when $N$ is a (prime) submodule of $M$. In the final section, we do the case when $N_{\Lambda}$ is not a submodule of $M$, and we improve Theorem 3.3 in [7] and Theorem 4.3 in [1] by Theorem 14, and Theorem 3.12 in [5] by Theorem 15.

Let $\Gamma$ be a simple graph. We say that $\Gamma$ is totally disconnected if none of two vertices of $\Gamma$ are adjacent. A subgraph $\Gamma_{1}$ of $\Gamma$ is an induced subgraph if vertex set of $\Gamma_{1}$ is contained in vertex set of $\Gamma$ and two vertices of $\Gamma_{1}$ are adjacent if and only if they are adjacent in $\Gamma$. Throughout this paper, all subgraphs are induced. We say that two subgraphs $\Gamma_{1}$ and $\Gamma_{2}$ of $\Gamma$ are disjoint if $\Gamma_{1}$ and $\Gamma_{2}$ have no common vertices and no vertex of $\Gamma_{1}$ (resp., $\Gamma_{2}$ ) is adjacent (in $\Gamma$ ) to any vertex not in $\Gamma_{1}$ (resp., $\Gamma_{2}$ ). If vertex $x$ is an end point of edge $e$, then $x$ and $e$ are called incident. The degree of a vertex $x$ in a graph $\Gamma$, written $\operatorname{deg}_{\Gamma}(x)$ is the number of edges incident to $x$. Graph $\Gamma$ is called $k-$ regular if degree of each vertex of $\Gamma$ is $k$. For vertices $x$ and $y$ of $\Gamma$, we define $d(x, y)$ to be the length of the shortest path from $x$ to $y$ $(d(x, x)=0$ and $d(x, y)=\infty$ if there is no such path). The diameter of $\Gamma$ is $\operatorname{diam}(\Gamma)=\sup \{d(x, y) \mid x$ and $y$ are vertices of $\Gamma\}$. The girth of $\Gamma$, denoted by 
$\operatorname{gr}(\Gamma)$, is the length of a shortest cycle in $\Gamma(\operatorname{gr} r(\Gamma)=\infty$ if $\Gamma$ contains no cycles). We denote the complete graph on $n$ vertices by $K^{n}$ and the complete bipartite graph on $m$ and $n$ vertices by $K^{m, n}$. We will call a $K^{1, n}$, a stargraph. A graph $\Gamma$ is called vertex transitive if for every two vertices $x$ and $y$ there exists $\tau \in \operatorname{Aut}(\Gamma)$ such that $\tau(x)=y$.

Throughout, all rings $R$ are commutative with $1 \neq 0$, and $M$ is an R-module with at least one prime submodule . For $A \subseteq M$, let $A^{*}=A \backslash\{0\}$. As usual, $\mathbb{Z}$ and $\mathbb{Z}_{n}$ will denote the integers and integers modulo $n$, respectively.

\section{2. $M(L)$ AS A UNION OF PRIME SUBMODULES}

In this section, we consider the question of when for an R-module $M$, the set $M(L)$ is a union of prime submodules and determine a family of non-primeless R-modules. We refer the reader to [1] for some properties concerning $M(L)$. Throughout this section, $L$ is a proper submodule of $M$ over the commutative ring $R$. Let $M(L)=$ $\left\{m \in M \mid r m \in L\right.$ for some $\left.r \in R \backslash\left(L:_{R} M\right)\right\}$. It is easy to see that $M(L)$ is closed under the multiplication of scalars. However $M(L)$ may not be an additive subgroup of $M$. Let $M=M(L)$, then $M(L)$ may or may not be a union of prime submodules. Also let $M(L)$ be a proper submodule of $M$, then it is a prime submodule of $M$, by [1, Theorem 2.1]. For our main result in this section, we need to the below theorem.

Theorem 1. Let $M$ be an $R$-module with $M \neq M(L)$, and let $\mathcal{A}=\left\{L_{\Delta} \mid L_{\Delta}\right.$ is a submodule of $M$ with $L_{\Delta} \subseteq M(L)$, and $L_{\Delta}=\bigcup_{h \in \Delta}\left(L:_{M} h\right)$ for some $\left.\Delta \subset R\right\}$. Then a maximal element of $\mathcal{A}$ is a prime submodule.

Proof. Let $L_{\Delta}=\bigcup_{h \in \Delta}\left(L:_{M} h\right)$ be a maximal element of $\mathcal{A}$. Suppose that $r m \in$ $L_{\Delta}$ for some $r \in R$ and $m \in M$ such that $m \notin L_{\Delta}$. First, assume that $r h \in R \backslash$ $\left(L:_{R} M\right)$ for every $h \in \Delta$. So each $\left(L:_{M} h\right) \subseteq\left(L:_{M} r h\right)$ and hence $L_{\Delta} \subseteq L_{\Delta}^{\prime}=$ $\bigcup_{h \in \Delta}(L: M r h)$. Also, suppose $l_{1}, l_{2} \in L_{\Delta}^{\prime}$. Then $l_{i} \in\left(L:_{M} r h_{i}\right)$ for $i=1,2$. So $r l_{i} \in\left(L:_{M} h_{i}\right) \subseteq L_{\Delta}$ and hence $r l_{1}+r l_{2} \in L_{\Delta}$. Thus $r l_{1}+r l_{2} \in\left(L:_{M} h_{j}\right)$ for some $h_{j} \in \Delta$; so $l_{1}+l_{2} \in\left(L:_{M} r h_{j}\right) \subseteq L_{\Delta}^{\prime}$. Since $L_{\Delta}^{\prime}$ is clearly closed under scalar product, $L_{\Delta}^{\prime}$ is submodule of $M$ with $L_{\Delta}^{\prime} \subseteq M(L)$. Thus by the maximality of $L_{\Delta}, L_{\Delta}=L_{\Delta}^{\prime}$. Now $r m \in L_{\Delta}$ implies $r m \in(L: M h)$ for some $h \in \Delta$. Hence $m \in\left(L:_{M} r h\right) \subseteq L_{\Delta}^{\prime}=L_{\Delta} ;$ a contradiction. Thus $r h \in\left(L:_{R} M\right)$ for some $h \in \Delta$ and hence $r M \subseteq\left(L:_{M} h\right) \subseteq L_{\Delta}$. Therefore $L_{\Delta}$ is a prime submodule of $M$.

Theorem 2. Let $M$ be an $R$-module with $M \neq M(L)$, then $M(L)$ is a union of prime submodules.

Proof. Let $l \in M(L), \mathcal{A}_{l}=\left\{L_{\Delta} \mid L_{\Delta}\right.$ is a submodule of $M, l \in L_{\Delta} \subseteq M(L)$, and $L_{\Delta}=\bigcup_{h \in \Delta}\left(L:_{M} h\right)$ for some $\left.\Delta \subset R\right\}$, and $r l \in L$ where $r \in R \backslash\left(L:_{R} M\right)$. Then $l \in\left(L:_{M} r\right)$; thus $\mathcal{A}_{l} \neq \varnothing$. By Zorn's Lemma, $\mathcal{A}_{l}$ has a maximal element $L_{l}$. By above theorem, $L_{l}$ is a prime submodule of $M(L)$. Therefore $M(L)=\bigcup_{l \in M(L)} L_{l}$ is a union of prime submodules of $M$. 
In the next corollary, we determine a family of non-primeless R-modules.

Corollary 1. Let $M$ be an $R$-module such that $M \neq M(L)$ for some proper submodule $L$ of $M$, then $M$ is not primeless.

Proof. This is clear from above theorem.

\section{BASIC PROPERTIES OF THE EXTENDED TOTAL GRAPH}

The basic properties of the extended total graph are given below, independent of whether or not $N_{\Lambda}$ is a submodule of $M$. Since $(c+1)(c-1)=0 \in H_{\Lambda}=\bigcup_{\lambda \in \Lambda} P_{\lambda}$, either $c+1 \in H_{\Lambda}$ or $c-1 \in H_{\Lambda}$. First, we determine properties of $T \Gamma_{c}\left(M, N_{\Lambda}\right)$ for some $c \in U(R)$.

Theorem 3. Let there is $c \in U(R)$ where $c \neq 1,-1$ and $c^{2}=1$.

(1) If $x+y \in N_{\Lambda}$, then either $x+c y \in N_{\Lambda}$ or $x-c y \in N_{\Lambda}$.

(2) If $c+1 \notin H_{\Lambda}$, then $T \Gamma_{c}\left(M, N_{\Lambda}\right) \cong T \Gamma_{1}\left(M, N_{\Lambda}\right)$.

(3) If $c-1 \notin H_{\Lambda}$, then $T \Gamma_{c}\left(M, N_{\Lambda}\right) \cong T \Gamma_{-1}\left(M, N_{\Lambda}\right)$.

Proof. (1) Let $x+y \in N_{\lambda}$ for some $\lambda \in \Lambda$, then $(c-1)(x-c y) \in N_{\lambda}$. Let $x-c y \notin N_{\lambda}$, then $c-1 \in P_{\lambda}$ hence $x+y+(c-1) y=x+c y \in N_{\lambda}$.

(2) Let $c+1 \notin H_{\Lambda}$ and $x+y \in N_{\Lambda}$, then $x+c y \in N_{\Lambda}$ since $(c+1)(x+c y) \in$ $N_{\Lambda}$. Also if $x+c y \in N_{\Lambda}$, then $x+y \in N_{\Lambda}$, since $(c+1)(x+y) \in N_{\Lambda}$ and $c+1 \notin H_{\Lambda}$.

(3) Let $c-1 \notin H_{\Lambda}$ and $x-y \in N_{\Lambda}$, then $x+c y \in N_{\Lambda}$ since $(c-1)(x+c y) \in$ $N_{\Lambda}$. Also if $x+c y \in N_{\Lambda}$, then $x-y \in N_{\Lambda}$, since $(c-1)(x-y) \in N_{\Lambda}$ and $c-1 \notin H_{\Lambda}$.

Remark 1. Suppose that $x, y \in M$ are adjacent in $T \Gamma_{1}\left(M, N_{\Lambda}\right)$, then $x$ and $c y$ are adjacent in $T \Gamma_{c}\left(M, N_{\Lambda}\right)$, if $x \neq c y$.

Theorem 4. Let $M$ be an R-module with $\left|N_{\Lambda}\right|=\alpha$ and $m \in M$. If $(c+1) m \notin N_{\Lambda}$, then $\operatorname{deg}_{T \Gamma_{c}\left(M, N_{\Lambda}\right)}(m)=\alpha$; otherwise, $\operatorname{deg}_{T \Gamma_{c}\left(M, N_{\Lambda}\right)}(m)=\alpha-1$. In particular, $c+1 \in H_{\Lambda}$ if and only if $T \Gamma_{c}\left(M, N_{\Lambda}\right)$ is a $(\alpha-1)$-regular graph.

Proof. There is a unique $x=n-\mathrm{cm} \in M$ for every $n \in N_{\Lambda}$. Hence $m$ is adjacent to $x$ unless $x=m$. If $(c+1) m \notin N_{\Lambda}$, then $n-c m \neq m$ for every $n \in N_{\Lambda}$. Therefore $\operatorname{deg}_{T \Gamma_{c}\left(M, N_{\Lambda}\right)}(m)=\alpha$. Otherwise, let $(c+1) m \in N_{\Lambda}$, then $\operatorname{deg}_{T \Gamma_{c}\left(M, N_{\Lambda}\right)}(m)=$ $\alpha-1$ since $m$ cannot be adjacent to itself. In particular, if $c+1 \in H_{\Lambda}$, then $(c+$ 1) $m \in N_{\Lambda}$ and $\operatorname{deg}_{T \Gamma_{c}\left(M, N_{\Lambda}\right)}(m)=\alpha-1$ for every $m \in M$. Now suppose that $T \Gamma_{c}\left(M, N_{\Lambda}\right)$ is a $(\alpha-1)$-regular graph, $c+1 \notin H_{\Lambda}$ and $m^{\prime} \in M \backslash N_{\Lambda}$, then $\operatorname{deg}_{T \Gamma_{c}\left(M, N_{\Lambda}\right)}\left(m^{\prime}\right)=\alpha$ since $(c+1) m^{\prime} \notin N_{\Lambda}$ for every $m^{\prime} \in M \backslash N_{\Lambda}$, a contradiction. so $c+1 \in H_{\Lambda}$.

Theorem 5. If $c-1 \notin H_{\Lambda}$, then $T \Gamma_{c}\left(M, N_{\Lambda}\right)$ is vertex transitive. 
Proof. For each $m \in M$ the mapping $\tau_{m}: x \longmapsto x+m$ is a permutation of the elements of $M$. Let $c-1 \notin H_{\Lambda}$, then $x+c y \in N_{\Lambda}$ if and only if $x+m+c(y+$ $m) \in N_{\Lambda}$ since $(c-1)(x+m+c(y+m))=(c-1)(x+c y) \in N_{\Lambda}$. Hence $\tau_{m}$ is an automorphism of $T \Gamma_{c}\left(M, N_{\Lambda}\right)$. The permutations $\tau_{m}$ form a subgroup of the automorphism group of $T \Gamma_{c}\left(M, N_{\Lambda}\right)$. This subgroup acts transitively on the vertices of $T \Gamma_{c}\left(M, N_{\Lambda}\right)$ because for any two vertices $m$ and $m^{\prime}$, the automorphism $\tau_{m-m^{\prime}}$ maps $m$ to $m^{\prime}$.

The previous theorem gave an important property of $T \Gamma_{c}\left(M, N_{\Lambda}\right)$. The vertex transitivity identifies some properties of graph such as the edge connectivity. For further investigation on vertex transitivity and edge connectivity, see [8]. Hence if $c-1 \notin H_{\Lambda}$ and $T \Gamma_{c}\left(M, N_{\Lambda}\right)$ is connected (i.e., $M=<N_{\Lambda}>$ by Theorem 12), then the edge connectivity of $T \Gamma_{c}\left(M, N_{\Lambda}\right)$ is equal to $\alpha-1$.

Example 1. Let $M=\mathbb{Z}_{3} \times \mathbb{Z}_{3}, R=\mathbb{Z}_{12}, c=5, N_{1}=\mathbb{Z}_{3} \times 0, N_{2}=0 \times \mathbb{Z}_{3}$, and $N_{\Lambda}=N_{1} \cup N_{2}$, then by Theorem 3(3), $T \Gamma_{5}\left(M, N_{\Lambda}\right) \cong T \Gamma_{11}\left(M, N_{\Lambda}\right) . T \Gamma_{5}\left(M, N_{\Lambda}\right)$ is a 4-regular graph by Theorem 4. Also, by Theorem $5, T \Gamma_{5}\left(M, N_{\Lambda}\right)$ is vertex transitive.

\section{THE CASE WHEN $N_{\Lambda}$ IS A SUbMOdULE OF $M$}

We know that $N_{\Lambda}=\bigcup_{\lambda \in \Lambda} N_{\lambda}$ is a proper submodule of $M$ if and only if $N_{\Lambda}$ is a prime submodule of $M$. Moreover $P_{\lambda}=\left(N_{\lambda}:_{R} M\right)$ and $H_{\Lambda}=\left(N_{\Lambda}:_{R} M\right)$ are prime ideals of $R$.

Theorem 6. Let $M$ be an R-module such that $N_{\Lambda}$ is a proper submodule of $M$. Then $T \Gamma_{c}\left(N_{\Lambda}, N_{\Lambda}\right)$ is a complete subgraph of $T \Gamma_{c}\left(M, N_{\Lambda}\right)$ and $T \Gamma_{c}\left(N_{\Lambda}, N_{\Lambda}\right)$ is disjoint from $T \Gamma_{c}\left(M \backslash N_{\Lambda}, N_{\Lambda}\right)$.

Proof. It is clear that $T \Gamma_{c}\left(N_{\Lambda}, N_{\Lambda}\right)$ is a complete subgraph of $T \Gamma_{c}\left(M, N_{\Lambda}\right)$. Suppose that $y \in M \backslash N_{\Lambda}$ and $x \in N_{\Lambda}$ are adjacent. Then $x+c y \in N_{\Lambda}$ and $c y \in N_{\Lambda}$ hence $c \in H_{\Lambda}$ which is a contradiction since $c \in U(R)$. Therefore, $T \Gamma_{c}\left(N_{\Lambda}, N_{\Lambda}\right)$ is disjoint from $T \Gamma_{c}\left(M \backslash N_{\Lambda}, N_{\Lambda}\right)$.

Definition 1. By a slice of $T \Gamma_{c}\left(M, N_{\Lambda}\right)$ we mean a subgraph of $T \Gamma_{c}\left(M, N_{\Lambda}\right)$ with a vertex set as the form $x+N_{\Lambda}$, denoted by $S_{x}$, for some $x \in M$.

It is easy to see that a slice of $T \Gamma_{c}\left(M, N_{\Lambda}\right)$ is an induced subgraph. So, two slices $S_{x}$ and $S_{y}$ are the same is and only if $x-y \in N_{\Lambda}$.

The next theorem gives a complete description of $T \Gamma_{c}\left(M, N_{\Lambda}\right)$. It also shows that non-isomorphic modules may have isomorphic total graphs. We allow $\alpha$ and $\beta$ to be infinite cardinals.

Theorem 7. Let $M$ be an R-module such that $N_{\Lambda}$ is a proper submodule of $M$, and let $H_{\Lambda}=\left(N_{\Lambda}:_{R} M\right),\left|N_{\Lambda}\right|=\alpha$ and $\left|M / N_{\Lambda}\right|=\beta$.

(1) If $c+1 \in H_{\Lambda}$, then $T \Gamma_{c}\left(M \backslash N_{\Lambda}, N_{\Lambda}\right)$ is the union of $\beta-1$ disjoint $K^{\alpha}$ 's. 
(2) If $c+1 \notin H_{\Lambda}$, then $T \Gamma_{c}\left(M \backslash N_{\Lambda}, N_{\Lambda}\right)$ is the union of $(\beta-1) / 2$ disjoint $K^{\alpha, \alpha}$ 's.

Proof. (1) Assume that $c+1 \in H_{\Lambda}$, and let $x \in M \backslash N_{\Lambda}$. Then the slice $S_{x}$ is a complete subgraph of $T \Gamma_{c}\left(M \backslash N_{\Lambda}, N_{\Lambda}\right)$ since $\left(x+n_{1}\right)+c\left(x+n_{2}\right)=$ $(c+1) x+n_{1}+n_{2} \in N_{\Lambda}$ for all $n_{1}, n_{2} \in N_{\Lambda}$ since $c+1 \in H_{\Lambda}$ and $N_{\Lambda}$ is a submodule of $M$. Note that distinct slices form disjoint subgraphs of $T \Gamma_{c}\left(M \backslash N_{\Lambda}, N_{\Lambda}\right)$ since if $x+n_{1}$ and $y+n_{2}$ are adjacent for some $y \in M \backslash$ $N_{\Lambda}$ and $n_{1}, n_{2} \in N_{\Lambda}$, then $x+c y=\left(x+n_{1}\right)+c\left(y+n_{2}\right)-\left(n_{1}+c n_{2}\right) \in N_{\Lambda}$ if and only if $x-y=(x+c y)-(c+1) y \in N_{\Lambda}$ since $c+1 \in H_{\Lambda}$ and $N_{\Lambda}$ is a submodule of $M$. Then $S_{x}=S_{y}$, a contradiction. Thus $T \Gamma_{c}\left(M \backslash N_{\Lambda}, N_{\Lambda}\right)$ is the union of $\beta-1$ disjoint subgraphs $S_{x}$, each of which is a $K^{\alpha}$, where $\alpha=\left|N_{\Lambda}\right|=\left|x+N_{\Lambda}\right|$.

(2) Assume that $c+1 \notin H_{\Lambda}$, and let $x \in M \backslash N_{\Lambda}$. Then no two distinct elements in $S_{x}$ are adjacent, suppose not. So, $\left(x+n_{1}\right)+c\left(x+n_{2}\right) \in N_{\Lambda}$ for $n_{1}, n_{2} \in$ $N_{\Lambda}$. This implies that $(c+1) x \in N_{\Lambda}$ hence $c+1 \in H_{\Lambda}$ since $N_{\Lambda}$ is a prime submodule of $M$, a contradiction. On the other hand, two slices $S_{x}$ and $S_{-c x}$ are disjoint and each vertex of $S_{-c x}$ is adjacent to all vertex of $S_{x}$. Thus $S_{x} \cup$ $S_{-c x}$ is a complete bipartite subgraph of $T \Gamma_{c}\left(M \backslash N_{\Lambda}, N_{\Lambda}\right)$. Furthermore, if $x+n_{1}$ is adjacent to $y+n_{2}$ for some $y \in M \backslash N_{\Lambda}$ and $n_{1}, n_{2} \in N_{\Lambda}$, then $y+c x \in N_{\Lambda}$ as in part(1) above, and hence $y+N_{\Lambda}=-c x+N_{\Lambda}$. Thus $T \Gamma_{c}\left(M \backslash N_{\Lambda}, N_{\Lambda}\right)$ is the union of $(\beta-1) / 2$ disjoint subgraphs $S_{x} \cup S_{-c x}$, each of which is $K^{\alpha, \alpha}$, where $\alpha=\left|N_{\Lambda}\right|=\left|x+N_{\Lambda}\right|$

From the above theorem, one can easily deduce when $T \Gamma_{c}\left(M \backslash N_{\Lambda}, N_{\Lambda}\right)$ is a complete or connected graph. The next theorem determines when $T \Gamma_{c}\left(M \backslash N_{\Lambda}, N_{\Lambda}\right)$ is either complete or connected.

Theorem 8. Let $M$ be an R-module such that $N_{\Lambda}$ is a proper submodule of $M$, and let $H_{\Lambda}=\left(N_{\Lambda}:_{R} M\right)$.

(1) Let $T \Gamma_{c}\left(M \backslash N_{\Lambda}, N_{\Lambda}\right)$ be a complete graph, then either $\left|M / N_{\Lambda}\right|=|M|=3$ or $\left|M / N_{\Lambda}\right|=2$. Its converse is true when either $\left|M / N_{\Lambda}\right|=|M|=3$ and $c+1 \notin\left(0:_{R} M\right)$ or $\left|M / N_{\Lambda}\right|=2$.

(2) Let $T \Gamma_{c}\left(M \backslash N_{\Lambda}, N_{\Lambda}\right)$ be a connected graph, then either $\left|M / N_{\Lambda}\right|=3$ or $\left|M / N_{\Lambda}\right|=2$. Its converse is true when either $\left|M / N_{\Lambda}\right|=3$ and $c-1 \in H_{\Lambda}$ or $\left|M / N_{\Lambda}\right|=2$.

(3) $T \Gamma_{c}\left(M \backslash N_{\Lambda}, N_{\Lambda}\right)$ (and hence $T \Gamma_{c}\left(N_{\Lambda}, N_{\Lambda}\right)$ and $T \Gamma_{c}\left(M, N_{\Lambda}\right)$ ) is a totally disconnected graph if and only if $N_{\Lambda}=\{0\}$ and $c+1 \in\left(0:_{R} M\right)$.

Proof. Let $\left|M / N_{\Lambda}\right|=\beta$ and $\alpha=\left|N_{\Lambda}\right|$

(1) Let $T \Gamma_{c}\left(M \backslash N_{\Lambda}, N_{\Lambda}\right)$ be a complete graph. Then by Theorem 7, $T \Gamma_{c}(M \backslash$ $N_{\Lambda}, N_{\Lambda}$ ) is a single $K^{\alpha}$ or $K^{1,1}$. If $c+1 \in H_{\Lambda}$, then $\beta-1=1$ thus $\beta=2$, 
and hence $\left|M / N_{\Lambda}\right|=2$. If $c+1 \notin H_{\Lambda}$, then $\alpha=1$ and $(\beta-1) / 2=1$. Thus $N_{\Lambda}=\{0\}$ and $\beta=3$; hence $\left|M / N_{\Lambda}\right|=|M|=3$. Conversely, suppose first that $M / N_{\Lambda}=\left\{N_{\Lambda}, x+N_{\Lambda}\right\}$, where $x \notin N_{\Lambda}$. Since $c x \notin N_{\Lambda}$, so, $x+N_{\Lambda}=$ $-c x+N_{\Lambda}$ which implies that $(c+1) x \in N_{\Lambda}$. Let $m, m^{\prime} \in M \backslash N_{\Lambda}$. Then $m+x, m^{\prime}+x \in N_{\Lambda}$ since none of $m+x+N_{\Lambda}$ and $m^{\prime}+x+N_{\Lambda}$ are equal to $x+N_{\Lambda}$ so $m+c m^{\prime}=(m+x)+c\left(m^{\prime}+x\right)-(c+1) x \in N_{\Lambda}$ since $N_{\Lambda}$ is a submodule of $M$. Thus $T \Gamma_{c}\left(M \backslash N_{\Lambda}, N_{\Lambda}\right)$ is complete. Next, suppose that $\left|M / N_{\Lambda}\right|=|M|=3$; hence $H_{\Lambda}=\left(0:_{R} M\right)$ since $N_{\Lambda}=\{0\}$. By Theorem $7, T \Gamma_{c}\left(M \backslash N_{\Lambda}, N_{\Lambda}\right)$ is a complete graph since $c+1 \notin\left(0:_{R} M\right)$.

(2) Let $T \Gamma_{c}\left(M \backslash N_{\Lambda}, N_{\Lambda}\right)$ be connected. Then by Theorem 7, $T \Gamma_{c}\left(M \backslash N_{\Lambda}, N_{\Lambda}\right)$ is a single $K^{\alpha}$ or $K^{\alpha, \alpha}$. If $c+1 \in H_{\Lambda}$, then $\beta-1=1$, and hence $\left|M / N_{\Lambda}\right|=$ 2. If $c+1 \notin H_{\Lambda}$, then $(\beta-1) / 2=1$, and hence $\left|M / N_{\Lambda}\right|=3$. Conversely, by part (1) above if $\left|M / N_{\Lambda}\right|=2$ then $T \Gamma_{c}\left(M \backslash N_{\Lambda}, N_{\Lambda}\right)$ is complete and so it is connected. Suppose that $\left|M / N_{\Lambda}\right|=3$ and $c-1 \in H_{\Lambda}$. First, we show that $c+1 \notin H_{\Lambda}$. Suppose not. Let $c+1 \in H_{\Lambda}$ and $M / N_{\Lambda}=$ $\left\{N_{\Lambda}, x+N_{\Lambda}, y+N_{\Lambda}\right\}$, where $x, y \notin N_{\Lambda}$; it is easy to see that $x+c y \in N$. This yields that $x$ and $y$ are adjacent, a contradiction, by the proof of Theorem 7(1). Thus $c+1 \notin H_{\Lambda}$. Therefore, $T \Gamma_{c}\left(M \backslash N_{\Lambda}, N_{\Lambda}\right)$ is the complete bipartite graph $K^{\alpha, \alpha}$, by Theorem 7(2).

(3) $T \Gamma_{c}\left(M \backslash N_{\Lambda}, N_{\Lambda}\right)$ is totally disconnected if and only if it be a disjoint union of $K^{1}$ 's. so by Theorem $7,\left|N_{\Lambda}\right|=1$. Further, since $m$ and $-\mathrm{cm}$ are adjacent for all $m \in M$, it follows that $m=-c m$, hence $c+1 \in\left(0:_{R} M\right)$.

The next theorem gives a more explicit description of the diameter of $T \Gamma_{c}(M \backslash$ $N_{\Lambda}, N_{\Lambda}$ ) when $N_{\Lambda}$ is a proper submodule of $M$.

Theorem 9. Let $M$ be an R-module such that $N_{\Lambda}$ is a proper submodule of $M$, and let $H_{\Lambda}=\left(N_{\Lambda}:_{R} M\right)$.

(1) $\operatorname{diam}\left(T \Gamma_{c}\left(M \backslash N_{\Lambda}, N_{\Lambda}\right)\right)=0$ if and only if $|M|=2$.

(2) $\operatorname{diam}\left(T \Gamma_{c}\left(M \backslash N_{\Lambda}, N_{\Lambda}\right)\right)=1$ if and only if either $N_{\Lambda} \neq\{0\}$ and $\left|M / N_{\Lambda}\right|=$ 2 or $|M|=3$ and $c+1 \notin(0: R M)$.

(3) $\left.\operatorname{diamT} \Gamma_{c}\left(M \backslash N_{\Lambda}, N_{\Lambda}\right)\right)=2$ if and only if $N_{\Lambda} \neq\{0\},\left|M / N_{\Lambda}\right|=3$ and $c-1 \in H_{\Lambda}$.

(4) Otherwise, $\operatorname{diam}\left(T \Gamma_{c}\left(M \backslash N_{\Lambda}, N_{\Lambda}\right)\right)=\infty$.

Proof. It is clear by the proof of the Theorem 8 .

The next theorem describes the girth of $T \Gamma_{c}\left(M \backslash N_{\Lambda}, N_{\Lambda}\right)$ and $T \Gamma_{c}\left(M, N_{\Lambda}\right)$ when $N_{\Lambda}$ is a proper submodule of $M$.

Theorem 10. Let $M$ be an R-module such that $N_{\Lambda}$ is a proper submodule of $M$, and let $H_{\Lambda}=\left(N_{\Lambda}:_{R} M\right)$.

(1) (a) $\operatorname{gr}\left(T \Gamma_{c}\left(M \backslash N_{\Lambda}, N_{\Lambda}\right)\right)=3$ if and only if $c+1 \in H_{\Lambda}$ and $\left|N_{\Lambda}\right| \geq 3$. 
(b) $\operatorname{gr}\left(T \Gamma_{c}\left(M \backslash N_{\Lambda}, N_{\Lambda}\right)\right)=4$ if and only if $c+1 \notin H_{\Lambda}$ and $\left|N_{\Lambda}\right| \geq 2$.

(c) Otherwise, $\operatorname{gr}\left(T \Gamma_{c}\left(M \backslash N_{\Lambda}, N_{\Lambda}\right)\right)=\infty$.

(2) (a) $\operatorname{gr}\left(T \Gamma_{c}\left(M, N_{\Lambda}\right)\right)=3$ if and only if $\left|N_{\Lambda}\right| \geq 3$.

(b) $\operatorname{gr}\left(T \Gamma_{c}\left(M, N_{\Lambda}\right)\right)=4$ if and only if $c+1 \notin H_{\Lambda}$ and $\left|N_{\Lambda}\right|=2$.

(c) Otherwise, $\operatorname{gr}\left(T \Gamma_{c}\left(M, N_{\Lambda}\right)\right)=\infty$.

Proof. Apply Theorem 7 and Theorem 6.

Example 2. Let $M=\mathbb{Z}_{2} \times \mathbb{Z}_{3}, R=\mathbb{Z}_{12}$ and $N_{\Lambda}=\mathbb{Z}_{2} \times 0$, then $T \Gamma_{c}(M)$ $\left.N_{\Lambda}, N_{\Lambda}\right)$ is the union of 2 disjoint $K^{2}$ 's, if $c=5$ or 11 , by Theorem 7(1) and $T \Gamma_{c}\left(M \backslash N_{\Lambda}, N_{\Lambda}\right)$ is a $K^{2,2}$, if $c=1$ or 7 , by Theorem 7(2). Now, if $c=1$ or 7, then $T \Gamma_{c}\left(M \backslash N_{\Lambda}, N_{\Lambda}\right)$ is connected with $\left.\operatorname{diam} T \Gamma_{c}\left(M \backslash N_{\Lambda}, N_{\Lambda}\right)\right)=2$ and $\operatorname{gr}\left(T \Gamma_{c}\left(M \backslash N_{\Lambda}, N_{\Lambda}\right)\right)=4$ by Theorem 8(2), 9(3) and 10(1)(b), respectively, and $\operatorname{gr}\left(T \Gamma_{c}\left(M, N_{\Lambda}\right)\right)=4$ by Theorem 10(2)(b).

\section{The CASE When $N_{\Lambda}$ IS NOT A Submodule of $M$}

In this section, we consider the remaining case when $N_{\Lambda}$ is not a submodule of $M$, this implies that $\left|N_{\Lambda}\right| \geq 3$. So there are distinct $x, y \in N_{\Lambda}^{*}$ such that $x+c y \in M \backslash N_{\Lambda}$. In this case, we show that $T \Gamma_{c}\left(N_{\Lambda}, N_{\Lambda}\right)$ is always connected but never complete. Moreover $T \Gamma_{c}\left(N_{\Lambda}, N_{\Lambda}\right)$ and $T \Gamma_{c}\left(M \backslash N_{\Lambda}, N_{\Lambda}\right)$ are never disjoint subgraphs of $T \Gamma_{c}\left(M, N_{\Lambda}\right)$. We first show that $T \Gamma_{c}\left(M, N_{\Lambda}\right)$ is connected when $T \Gamma_{c}\left(M \backslash N_{\Lambda}, N_{\Lambda}\right)$ is connected. However, we give an example to show that the converse fails.

Theorem 11. Let $M$ be an R-module and $N_{\Lambda}$ a union of prime submodules of $M$ that is not a submodule of $M$.

(1) $T \Gamma_{c}\left(N_{\Lambda}, N_{\Lambda}\right)$ is connected with diam $\left(T \Gamma_{c}\left(N_{\Lambda}, N_{\Lambda}\right)\right)=2$.

(2) Some vertices of $T \Gamma_{c}\left(N_{\Lambda}, N_{\Lambda}\right)$ are adjacent to a vertex of $T \Gamma_{c}\left(M \backslash N_{\Lambda}, N_{\Lambda}\right)$. In particular, the subgraphs $T \Gamma_{c}\left(N_{\Lambda}, N_{\Lambda}\right)$ and $T \Gamma_{c}\left(M \backslash N_{\Lambda}, N_{\Lambda}\right)$ of $T \Gamma_{c}\left(M, N_{\Lambda}\right)$ are not disjoint.

(3) If $T \Gamma_{c}\left(M \backslash N_{\Lambda}, N_{\Lambda}\right)$ is connected, then $T \Gamma_{c}\left(M, N_{\Lambda}\right)$ is connected.

Proof. (1) Every $x \in N_{\Lambda}^{*}$ is adjacent to 0 . Thus $x-0-y$ is a path in $T \Gamma_{c}\left(N_{\Lambda}, N_{\Lambda}\right)$ of length two between any two distinct $x, y \in N_{\Lambda}^{*}$. Moreover, there are nonadjacent $x, y \in N_{\Lambda}^{*}$ since $N_{\Lambda}$ is not a submodule of $M$; so $\operatorname{diam}\left(T \Gamma_{c}\left(N_{\Lambda}, N_{\Lambda}\right)\right)=2$.

(2) Since $N_{\Lambda}$ is not a submodule of $M$, there are distinct $x, y \in N_{\Lambda}^{*}$ such that $x+c y \in M \backslash N_{\Lambda}$. Then $-y \in N_{\Lambda}^{*}$ and $x+c y \in M \backslash N_{\Lambda}$ are adjacent vertices in $T \Gamma_{c}\left(M, N_{\Lambda}\right)$ since $x+c y-c y=x \in N_{\Lambda}$. The "in particular" statement is clear.

(3) By part (1) above, it suffices to show that there is a path from $x$ to $y$ in $T \Gamma_{c}\left(M, N_{\Lambda}\right)$ for every $x \in N_{\Lambda}$ and $y \in M \backslash N_{\Lambda}$. By part (2) above, there are adjacent vertices $u$ and $v$ in $T \Gamma_{c}\left(N_{\Lambda}, N_{\Lambda}\right)$ and $T \Gamma_{c}\left(M \backslash N_{\Lambda}, N_{\Lambda}\right)$, respectively. Since $T \Gamma_{c}\left(N_{\Lambda}, N_{\Lambda}\right)$ is connected, there is a path from $x$ to 
$u$ in $T \Gamma_{c}\left(N_{\Lambda}, N_{\Lambda}\right)$; and since $T \Gamma_{c}\left(M \backslash N_{\Lambda}, N_{\Lambda}\right)$ is connected, there is a path from $v$ to $y$ in $T \Gamma_{c}\left(M \backslash N_{\Lambda}, N_{\Lambda}\right)$, then there is a path from $x$ to $y$ in $T \Gamma_{c}\left(M, N_{\Lambda}\right)$ since $u$ and $v$ are adjacent in $T \Gamma_{c}\left(M, N_{\Lambda}\right)$. Thus $T \Gamma_{c}\left(M, N_{\Lambda}\right)$ is connected.

Next, we determine an equivalent condition for connectedness of $T \Gamma_{c}\left(M, N_{\Lambda}\right)$ and compute $\operatorname{diam}\left(T \Gamma_{c}\left(M, N_{\Lambda}\right)\right)$. As usual, if $A \subseteq M$, then $\langle A>$ denotes the submodule of $M$ generated by $A$.

Theorem 12. Let $M$ be an R-module and $N_{\Lambda}$ a union of prime submodule of $M$. Then $T \Gamma_{c}\left(M, N_{\Lambda}\right)$ is connected if and only if $M=<N_{\Lambda}>$ (i.e., $m=n_{1}+n_{2}+$ $\cdots+n_{k}$ for every $m \in M$ and for some $n_{1}, \ldots, n_{k} \in N_{\Lambda}$ and $\left.k \in \mathbb{N}\right)$.

Proof. Suppose that $T \Gamma_{c}\left(M, N_{\Lambda}\right)$ is connected, let $m \in M$. Then there is a path $0-m_{1}-\cdots-m_{\iota}-m$ from 0 to $m$ in $T \Gamma_{c}\left(M, N_{\Lambda}\right)$. Thus $m_{1}, m_{2}+c m_{1}, \ldots, m+$ $c m_{\iota} \in N_{\Lambda}$. Hence $m \in<m_{1}, m_{2}+c m_{1}, \ldots, m+c m_{\iota}>\subseteq<N_{\Lambda}>$; thus $M=<N_{\Lambda}>$. Conversely, suppose that $M=<N_{\Lambda}>$. We show that there is a path from 0 to $m$ in $T \Gamma_{c}\left(M, N_{\Lambda}\right)$ for every $0 \neq m \in M$. By hypothesis, $m=n_{1}+n_{2}+\cdots+n_{k}$ for some $n_{1}, \ldots, n_{k} \in N_{\Lambda}$ and $k \in \mathbb{N}$. Also we define a function, denoted by $f(t)$, which is equal to 0 where $t$ is even and equal to 1 where $t$ is odd. Let $a_{0}=0$ and $a_{j}=(-1)^{f(k-j)} c^{f(k-j)}\left(n_{1}+\cdots+n_{j}\right)$ for every integer $j$ with $1 \leq j \leq k$. Then $a_{j}+c a_{j+1} \in N_{\Lambda}$ for every integer $j$ with $0 \leq j \leq k-1$, and thus $0=a_{0}-a_{1}-$ $\cdots-a_{k-1}-a_{k}=m$ is a path from 0 to $m$ in $T \Gamma_{c}\left(M, N_{\Lambda}\right)$ of length at most $k$. Now, let $0 \neq u, w \in M$. Then by the preceding argument, there are paths from $u$ to 0 and 0 to $w$ in $T \Gamma_{c}\left(M, N_{\Lambda}\right)$. Hence there is a path from $u$ to $w$ in $T \Gamma_{c}\left(M, N_{\Lambda}\right)$; so $T \Gamma_{c}\left(M, N_{\Lambda}\right)$ is connected.

In the next theorem and its corollary, we determine a relation between $T \Gamma_{c}\left(R, H_{\Lambda}\right)$ and $T \Gamma_{c}\left(M, N_{\Lambda}\right)$, where $H_{\Lambda}=\left(N_{\Lambda}:_{R} M\right)$.

Theorem 13. Let $M$ be an $R$-module, and $N_{\Lambda}$ a union of prime submodules of $M$ where $H_{\Lambda}=\left(N_{\Lambda}:_{R} M\right)$. If $T \Gamma_{c}\left(R, H_{\Lambda}\right)$ is connected, then $T \Gamma_{c}\left(M, N_{\Lambda}\right)$ is connected as well.

Proof. Suppose that $T \Gamma_{c}\left(R, H_{\Lambda}\right)$ is connected and let $m \in M$. Then there is a path $0-r_{1}-\cdots-r_{k}-1$ from 0 to 1 in $T \Gamma_{c}\left(R, H_{\Lambda}\right)$. Then $r_{1}, r_{2}+c r_{1}, \ldots, r_{k}+c r_{k-1}, 1+$ $c r_{k} \in H_{\Lambda}$. Hence $0-r_{1} m-\cdots-r_{k} m-m$ is a path from 0 to $m$ in $T \Gamma_{c}\left(M, N_{\Lambda}\right)$. Since all vertices may be connected via $0, T \Gamma_{c}\left(M, N_{\Lambda}\right)$ is a connected graph.

Corollary 2. Let $M$ be an R-module, and $N_{\Lambda}$ a union of prime submodules of $M$ and $H_{\Lambda}=\left(N_{\Lambda}:_{R} M\right)$. If $R=<H_{\Lambda}>$, then $T \Gamma_{c}\left(M, N_{\Lambda}\right)$ is connected.

Proof. This follows directly from Theorem 12 and 13.

Remark 2. According to the proof of the Theorem 13, if $d(0,1)=k$ in $T \Gamma_{c}\left(R, H_{\Lambda}\right)$, then $d(0, m) \leq k$ in $T \Gamma_{c}\left(M, N_{\Lambda}\right)$ for every $m \in M$. 
In next theorem, we improve Theorem 3.3 of [7] and Theorem 4.3 of [1] without cosidering $M$ as a finitely generated R-module.

Theorem 14. Let $M$ be an R-module and $N_{\Lambda}$ a union of prime submodule of $M$ that is not a submodule of $M$ such that $M=<N_{\Lambda}>$ (i.e., $T \Gamma_{c}\left(M, N_{\Lambda}\right)$ is connected). If there is $k \geq 2$ which is a greatest integer $i$ such that $m=n_{1}+n_{2}+$ $\cdots+n_{i}$ for every $m \in M$ and for some $n_{1}, \ldots, n_{i} \in N_{\Lambda}$ where $n_{1}+n_{2}+\cdots+n_{i}$ is a shortest representation of the element $m$, then $\operatorname{diam}\left(T \Gamma_{c}\left(M, N_{\Lambda}\right)\right)=k$. Otherwise, $\operatorname{diam}\left(T \Gamma_{c}\left(M, N_{\Lambda}\right)\right)=\infty$.

Proof. Let $m$ and $m^{\prime}$ be distinct elements in $M$ such that they are not adjacent. We show that there is a path from $m$ to $m^{\prime}$ in $T \Gamma_{c}\left(M, N_{\Lambda}\right)$ with length at most $k$. We define a function, denoted by $f(t)$, which is equal to 0 where $t$ is even and equal to 1 where $t$ is odd. By the proof of Theorem 12, we can consider two path $\left(m-m^{\prime}\right)-$ $x_{1}-\cdots-x_{k-1}-0$ and $\left(m+c m^{\prime}\right)-y_{1}-\cdots-y_{k-1}-0$ of lengths at most $k$. Let $a_{0}=$ $m, a_{k}=m^{\prime}, a_{j}=(-1)^{f(k-j)} c^{f(k-j)} m^{\prime}+x_{j} f(k-1)+y_{j} f(k)$ for every integers $j$ with $1 \leq j \leq k-1$. Then $a_{j}+c a_{j+1} \in N_{\Lambda}$ for every integers $j$ with $0 \leq j \leq k-1$, and thus $m=a_{0}-a_{1}-\cdots-a_{k-1}-a_{k}=m^{\prime}$ is a path from $m$ to $m^{\prime}$ in $T \Gamma_{c}\left(M, N_{\Lambda}\right)$ of length at most $k$. Now we show that any path from 0 to $m$ in $T \Gamma_{c}\left(M, N_{\Lambda}\right)$ has length at least $k$. Suppose that $0-b_{1}-\cdots-b_{\iota-1}-m$ is a path from 0 to $m$ in $T \Gamma_{c}\left(M, N_{\Lambda}\right)$ of length $\iota$ and let $m=n_{1}+\cdots+n_{k}$ be a shortest representation of the element $m$. Thus $b_{1}, b_{2}+c b_{1}, \ldots, b_{l-1}+c b_{l-2}, m+c b_{l-1} \in N_{\Lambda}$, and hence $m \in<$ $b_{1}, b_{2}+c b_{1}, \ldots, b_{l-1}+c b_{\iota-2}, m+c b_{l-1}>$. Thus $\iota \geq k$ and so the shortest path between 0 and $m$ in $T \Gamma_{c}\left(M, N_{\Lambda}\right)$ has length $k$. Therefore $\operatorname{diam}\left(T \Gamma_{c}\left(M, N_{\Lambda}\right)\right)=k$. If there is no such $k$, then we show that $\operatorname{diam}\left(T \Gamma_{c}\left(M, N_{\Lambda}\right)\right)=\infty$. Suppose not. Let $\operatorname{diam}\left(T \Gamma_{c}\left(M, N_{\Lambda}\right)\right)=t$ where $t<\infty$. Since $k=\infty$, there is $m \in M$ such that $m=n_{1}+\cdots+n_{t+1}$ is a shortest representation of the element $m$. According what is proved, it is contradiction since there is a path from 0 to $m$ in $T \Gamma_{c}\left(M, N_{\Lambda}\right)$ of length at most $t$.

Remark 3. Let $N_{\Lambda}=\bigcup_{\lambda \in \Lambda} N_{\lambda}$ and $M=<N_{\Lambda}>$, then it is clear that $1 \leq k \leq|\Lambda|$ where $k$ is as mentioned in Theorem 14.

Corollary 3. Let $M$ be an R-module and $N_{\Lambda}$ a union of prime submodule of $M$ such that $T \Gamma_{c}\left(M, N_{\Lambda}\right)$ is connected. Let $m=n_{1}+\cdots+n_{k}$ be a shortest representation of the element $m$ and $k$ is as mentioned in Theorem 14.

(1) $\operatorname{diam}\left(T \Gamma_{c}\left(M, N_{\Lambda}\right)\right)=d(0, m)$

(2) If $\operatorname{diam}\left(T \Gamma_{c}\left(M, N_{\Lambda}\right)\right)=k$ then $\operatorname{diam}\left(T \Gamma_{c}\left(M \backslash N_{\Lambda}, N_{\Lambda}\right)\right) \geq k-2$.

Proof. (1) This is clear from the proof of Theorem 14.

(2) Since $k=\operatorname{diam}\left(T \Gamma_{c}\left(M, N_{\Lambda}\right)\right)=d(0, m)$ by part (1) above, let $0-b_{1}-\cdots-$ $b_{k-1}-m$ be a shortest path from 0 to $m$ in $T \Gamma_{c}\left(M, N_{\Lambda}\right)$. Clearly $b_{1} \in N_{\Lambda}$. If $b_{i} \in N_{\Lambda}$ for some integer $i$ with $2 \leq i \leq k-1$, then the path $0-b_{i}-$ 
$\cdots-b_{k-1}-m$ from 0 to $m$ has length less than $k$, a contradiction. Thus $b_{i} \in M \backslash N_{\Lambda}$ for every integer $i$ with $2 \leq i \leq k-1$. Hence $b_{2}-\cdots-b_{k-1}-m$ is a shortest path from $b_{2}$ to $m$ in $T \Gamma_{c}\left(M \backslash N_{\Lambda}, N_{\Lambda}\right)$ of length $k-2$. Thus $\operatorname{diam}\left(T \Gamma_{c}\left(M \backslash N_{\Lambda}, N_{\Lambda}\right)\right) \geq k-2$.

We next investigate the girth of $T \Gamma_{c}\left(N_{\Lambda}, N_{\Lambda}\right), T \Gamma_{c}\left(M \backslash N_{\Lambda}, N_{\Lambda}\right)$, and $T \Gamma_{c}\left(M, N_{\Lambda}\right)$ when $N_{\Lambda}$ is not a submodule of $M$. Recall that $H_{\Lambda}=\left(N_{\Lambda}:_{R} M\right)=$ $\bigcup_{\lambda \in \Lambda} P_{\lambda}$ where $N_{\Lambda}$ is proper subset of $M$ with $\left|N_{\Lambda}\right| \geq 3$ and $P_{\lambda}=\left(N_{\lambda}: R M\right)$.

Theorem 15. Let $M$ be an R-module, and let $N_{\Lambda}=\bigcup_{\lambda} N_{\lambda}$ for prime submodules $N_{\lambda}$ of $M$, that is not a submodule of $M$ and $H_{\Lambda}=\left(N_{\Lambda}:_{R} M\right)$. Suppose that $m_{1}-m_{2}-m_{3}$ is a path of length two in $T \Gamma_{c}\left(M \backslash N_{\Lambda}, N_{\Lambda}\right)$ for distinct elements $m_{1}, m_{2}, m_{3} \in M \backslash N_{\Lambda}$.

(1) If $c+1 \in H_{\Lambda}$ and $\bigcap_{\lambda} N_{\lambda} \neq\{0\}$, then $\operatorname{gr}\left(T \Gamma_{c}\left(M \backslash N_{\Lambda}, N_{\Lambda}\right)\right)=3$.

(2) If $(c+1) m_{i} \neq 0$ for all integers $i$ with $1 \leq i \leq 3$, then $\operatorname{gr}\left(T \Gamma_{c}\left(M \backslash N_{\Lambda}, N_{\Lambda}\right)\right) \leq 4$.

Proof. (1) Suppose that there is a $0 \neq h \in \bigcap_{\lambda} N_{\lambda}$. If $m_{2} \neq m_{1}+h$, then $m_{1}-m_{2}-\left(m_{1}+h\right)-m_{1}$ is a cycle of length three in $T \Gamma_{c}\left(M \backslash N_{\Lambda}, N_{\Lambda}\right)$ since $(c+1) m_{1} \in N_{\Lambda}$. Hence, assume that $m_{2}=m_{1}+h$. Since $\left(m_{1}+h\right)+$ $c m_{3}=m_{2}+c m_{3} \in N_{\Lambda}$ and $h \in \bigcap_{\lambda} N_{\lambda}$, we have $m_{1}+c m_{3} \in N_{\Lambda}$. Thus $m_{1}-m_{2}-m_{3}-m_{1}$ is a cycle of length three in $T \Gamma_{c}\left(M \backslash N_{\Lambda}, N_{\Lambda}\right)$. Thus $\operatorname{gr}\left(T \Gamma_{c}\left(M \backslash N_{\Lambda}, N_{\Lambda}\right)\right)=3$.

(2) Suppose that $(c+1) m_{i} \neq 0$ for all integers $i$ with $1 \leq i \leq 3$. Then $m_{i} \neq$ $-c m_{i}$ for every $i$ with $1 \leq i \leq 3$. There are distinct integer $j, k$ with $1 \leq$ $j, k \leq 3$ such that they are adjacent and $m_{j} \neq-c m_{k}$ since if $m_{1}+c m_{2}=$ $m_{3}+c m_{2}=0$, then $m_{1}=m_{3}$, a contradiction. Thus $m_{j}-m_{k}-\left(-c m_{k}\right)-$ $\left(-c m_{j}\right)-m_{j}$ is a 4-cycle in $T \Gamma_{c}\left(M \backslash N_{\Lambda}, N_{\Lambda}\right)$; $\operatorname{sog} r\left(T \Gamma_{c}\left(M \backslash N_{\Lambda}, N_{\Lambda}\right)\right) \leq$ 4.

In the above theorem, we improve the proof of Theorem 3.12(1) in [5]. Also in the part (2) of the above theorem, $0 \neq(c+1) m_{i}$ can belongs to $N_{\Lambda}$ unlike Theorem $3.12(3)$ in [5].

Corollary 4. Let $M$ be an R-module, and let $N_{\Lambda}=\bigcup_{\lambda} N_{\lambda}$ for prime submodules $N_{\lambda}$ of $M$, that is not a submodule of $M$ and $H_{\Lambda}=\left(N_{\Lambda}:_{R} M\right)$. Suppose that $m_{1}-m_{2}-m_{3}$ is a path of length two in $T \Gamma_{c}\left(M \backslash N_{\Lambda}, N_{\Lambda}\right)$ for distinct elements $m_{1}, m_{2}, m_{3} \in M \backslash N_{\Lambda}$. If $(c+1) m_{i}=0$ for some integer $i$ with $1 \leq i \leq 3$ and $c+1 \notin\left(0:_{R} M\right)$, then $\operatorname{gr}\left(T \Gamma_{c}\left(M \backslash N_{\Lambda}, N_{\Lambda}\right)\right)=3$.

Proof. Suppose that $c+1 \notin\left(0:_{R} M\right)$. Thus $c+1 \neq 0$. Since $m_{i} \in M \backslash N_{\Lambda}$ for all integers $i$ with $1 \leq i \leq 3$ and $(c+1) m_{i}=0$ for some $i$ with $1 \leq i \leq 3$, we have $c+1 \in P_{\lambda}$ for every $P_{\lambda}$ where $P_{\lambda}=\left(N_{\lambda}: R M\right)$. Hence $0 \neq c+1 \in \bigcap_{\lambda} P_{\lambda}$. 
Since $c+1 \notin\left(0:_{R} M\right)$, there is a $m \in M$ such that $0 \neq(c+1) m \in \bigcap_{\lambda} N_{\lambda}$. Thus $\operatorname{gr}\left(T \Gamma_{c}\left(M \backslash N_{\Lambda}, N_{\Lambda}\right)\right)=3$ by Theorem 15(1).

Corollary 5. Let $M$ be an R-module, and let $N_{\Lambda}=\bigcup_{\lambda} N_{\lambda}$ for prime submodules $N_{\lambda}$ of $M$ and $H_{\Lambda}=\left(N_{\Lambda}:_{R} M\right)$. Suppose that $m_{1}-m_{2}-m_{3}$ is a path of length two in $T \Gamma_{c}\left(M \backslash N_{\Lambda}, N_{\Lambda}\right)$ for distinct elements $m_{1}, m_{2}, m_{3} \in M \backslash N_{\Lambda}, c+1 \in H_{\Lambda}$, $\bigcap_{\lambda} N_{\lambda} \neq\{0\}$ and $\left|N_{\Lambda}\right| \geq 3$, then $\operatorname{gr}\left(T \Gamma_{c}\left(M \backslash N_{\Lambda}, N_{\Lambda}\right)\right)=3$.

Proof. This follows directly from Theorem 10(1)(a) and Theorem 15(1).

Theorem 16. Let $M$ be an R-module and $N_{\Lambda}$ a union of prime submodule of $M$ that is not a submodule of $M$.

(1) Either $\operatorname{gr}\left(T \Gamma_{c}\left(N_{\Lambda}, N_{\Lambda}\right)\right)=3 \operatorname{org} r\left(T \Gamma_{c}\left(N_{\Lambda}, N_{\Lambda}\right)\right)=\infty$. Moreover, if one has $\operatorname{gr}\left(T \Gamma_{c}\left(N_{\Lambda}, N_{\Lambda}\right)\right)=\infty$, then $\left|N_{\lambda}\right|=2$ for any $\lambda \in \Lambda$ where $N_{\lambda}$ is a non-zero submodule of $M$. Also, $T \Gamma_{c}\left(N_{\Lambda}, N_{\Lambda}\right)$ is a star graph.

(2) $\operatorname{gr}\left(T \Gamma_{c}\left(M, N_{\Lambda}\right)\right)=3$ if and only if $g r\left(T \Gamma_{c}\left(N_{\Lambda}, N_{\Lambda}\right)\right)=3$.

(3) $\operatorname{gr}\left(T \Gamma_{c}\left(M, N_{\Lambda}\right)\right) \leq 4$.

(4) $\operatorname{gr}\left(T \Gamma_{c}\left(M, N_{\Lambda}\right)\right)=4$ if and only if $g r\left(T \Gamma_{c}\left(N_{\Lambda}, N_{\Lambda}\right)\right)=\infty$.

Proof. (1) If $n+c n^{\prime} \in N_{\Lambda}$ for some distinct $n, n^{\prime} \in N_{\Lambda}^{*}$, then $0-n-n^{\prime}-0$ is a 3-cycle in $T \Gamma_{c}\left(N_{\Lambda}, N_{\Lambda}\right)$; so $g r\left(T \Gamma_{c}\left(N_{\Lambda}, N_{\Lambda}\right)\right)=3$. Otherwise, $n+c n^{\prime} \in$ $M \backslash N_{\Lambda}$ for all distinct $n, n^{\prime} \in N_{\Lambda}^{*}$. So in this case, every $n \in N_{\Lambda}$ is adjacent to 0 , and no two distinct $n, n^{\prime} \in N_{\Lambda}^{*}$ are adjacent. Thus $T \Gamma_{c}\left(N_{\Lambda}, N_{\Lambda}\right)$ is a star graph with center 0 ; $\operatorname{sog} \operatorname{gr}\left(T \Gamma_{c}\left(N_{\Lambda}, N_{\Lambda}\right)\right)=\infty$. Moreover, let $N_{\Lambda}=$ $\bigcup_{\lambda \in \Lambda} N_{\lambda}$ is not a submodule of $M$ where $N_{\lambda}$ is a prime submodule of $M$ so $|\Lambda| \geq 2$. Assume that $\operatorname{gr}\left(T \Gamma_{c}\left(N_{\Lambda}, N_{\Lambda}\right)\right)=\infty$. Then $x+c y \in M \backslash N_{\Lambda}$ for all distinct $x, y \in N_{\Lambda}^{*}$, and thus if $N_{\lambda} \neq\{0\}$, then each $\left|N_{\lambda}\right|=2$.

(2) It suffices to show that $\operatorname{gr}\left(T \Gamma_{c}\left(N_{\Lambda}, N_{\Lambda}\right)\right)=3$ when $\operatorname{gr}\left(T \Gamma_{c}\left(M, N_{\Lambda}\right)\right)=3$. Let $(c+1) n \neq 0$ for some $n \in N_{\Lambda}^{*}$, then $0-n-(-c n)-0$ is a 3-cycle in $T \Gamma_{c}\left(N_{\Lambda}, N_{\Lambda}\right)$. Otherwise, $(c+1) n=0$ for all $n \in N_{\Lambda}$. Since $N_{\Lambda}$ is not a submodule of $M$, there are distinct elements $n, n^{\prime} \in N_{\Lambda}$ such that $n+n^{\prime} \in$ $M \backslash N_{\Lambda}$. Then $(c+1)\left(n+n^{\prime}\right)=0$, thus $c+1 \in H_{\Lambda}$. Let $m-m_{1}-m_{2}-m$ be a 3-cycle in $T \Gamma_{c}\left(M, N_{\Lambda}\right)$. Then $n_{1}=\mathrm{cm}+m_{1}, n_{2}=\mathrm{cm}+m_{2}, m_{1}+$ $c m_{2} \in N_{\Lambda}$. Thus $0-n_{1}-n_{2}-0$ is a 3-cycle in $T \Gamma_{c}\left(N_{\Lambda}, N_{\Lambda}\right)$; therefore $\operatorname{gr}\left(T \Gamma_{c}\left(N_{\Lambda}, N_{\Lambda}\right)\right)=3$.

(3) Since $N_{\Lambda}$ is not a submodule of $M$, there are distinct elements $n, n^{\prime} \in N_{\Lambda}$ such that $n+n^{\prime} \in M \backslash N_{\Lambda}$. Then $0-(-c n)-n+n^{\prime}-\left(-c n^{\prime}\right)-0$ is a 4-cycle in $T \Gamma_{c}\left(M, N_{\Lambda}\right)$.

(4) This follows by parts (1), (2) and (3) above.

Example 3. (a) Let $M=\mathbb{Z}[X]$ be an $\mathbb{Z}[X]$-module. Then $N_{\Lambda}=\mathbb{Z}[X] \backslash \mathbb{Z}^{*}$ is a union of prime submodule of $M$, that is not a submodule of $M$. Thus $T \Gamma_{c}\left(N_{\Lambda}, N_{\Lambda}\right)$ is connected with $\operatorname{diam}\left(T \Gamma_{c}\left(N_{\Lambda}, N_{\Lambda}\right)\right)=2$ by Theorem 
11(1). Moreover, by Theorem 12 and $14, T \Gamma_{c}\left(M, N_{\Lambda}\right)$ is connected with $\operatorname{diam}\left(T \Gamma_{c}\left(M, N_{\Lambda}\right)\right)=2$ since $z=X+z-X$ for $X, z-X \in N_{\Lambda}$ for every $z \in \mathbb{Z}^{*}$. However, $T \Gamma_{c}\left(M \backslash N_{\Lambda}, N_{\Lambda}\right)$ is not connected since there is no path from 1 to 2 in $T \Gamma_{c}\left(M \backslash N_{\Lambda}, N_{\Lambda}\right)$. Thus the converse of Theorem 11(3) need not hold.

(b) Let $k \in \mathbb{N}, M=\mathbb{Z}_{2} \times \mathbb{Z}_{2} \times \mathbb{Z}_{2}, R=\mathbb{Z}_{2 k}, N_{1}=\mathbb{Z}_{2} \times 0 \times 0, N_{2}=0 \times \mathbb{Z}_{2} \times 0$, $N_{3}=0 \times 0 \times \mathbb{Z}_{2}$ and $N_{\Lambda}=\bigcup N_{j}$ for $1 \leq j \leq 3$, then by Theorem 12 and 14 , $T \Gamma_{c}\left(M, N_{\Lambda}\right)$ is connected with $\operatorname{diam}\left(T \Gamma_{c}\left(M, N_{\Lambda}\right)\right)=3$, note that $(1,1,0)$ is the sum of two elements of $N_{\Lambda}$ but $(1,1,1)$ is the sum of three element of $N_{\Lambda}$, and $T \Gamma_{c}\left(R, H_{\Lambda}\right)$ is disconnected so the converse of Theorem 13 is fails, also $\operatorname{gr}\left(T \Gamma_{c}\left(N_{\Lambda}, N_{\Lambda}\right)\right)=\infty$ and $\operatorname{gr}\left(T \Gamma_{c}\left(M, N_{\Lambda}\right)\right)=4$ by Theorem 16.

\section{REFERENCES}

[1] A. Abbasi and S. Habibi, "The total graph of a module over a commutative ring with respect to proper submodules," J. Algebra Appl., vol. 11, no. 3, p. 13pp, 2012, doi: 10.1142/S0219498811005762.

[2] R. Akhtar, M. Boggess, T. Jackson-Henderson, I. Jimènez, R. Karpman, A. Kinzel, and D. Pritikin, "On the unitary Cayley graph of a finite ring," Electron. J. Combin., vol. 16, p. 13pp, 2009.

[3] D. D. Anderson and S. Chun, "The Set of Torsion Element of a Module," Comm. Algebra, vol. 42, no. 4, pp. 1835-1843, 2014, doi: 10.1080/00927872.2013.796555.

[4] D. F. Anderson and A. Badawi, "The total graph of a commutative ring," J. Algebra, vol. 320, no. 7, pp. 2706-2719, 2008, doi: 10.1016/j.jalgebra.2008.06.028.

[5] D. F. Anderson and A. Badawi, "The generalized total graph of a commutative ring," J. Algebra Appl., vol. 12, no. 5, p. 18pp, 2013, doi: 10.1142/S021949881250212X.

[6] N. Ashrafi, H. R. Maimani, M. R. Pournaki, and S. Yassemi, "Unit graphs associated with rings," Comm. Algebra, vol. 38, pp. 2851-2871, 2010, doi: 10.1080/00927870903095574.

[7] S. Ebrahimi Atani and S. Habibi, "The total torsion element graph of a module over a commutative ring," An. St. Univ. Ovidius Constanta, vol. 19, no. 1, pp. 23-34, 2011.

[8] C. Godsil and G. F. Royle, Algebraic Graph Theory. New York: Springer-Verlag, 2001. doi: 10.1007/978-1-4613-0163-9.

[9] C. Lanski and A. Maròti, "Rings elements as sums of units," Cent. Eur. J. Math., vol. 7, pp. 395-399, 2009, doi: 10.2478/s11533-009-0024-5.

[10] Z. Pucanovic, "The total graph of a module," Matematicki vesnik, vol. 63, no. 4, pp. 305-312, 2011.

Authors' addresses

A. Abbasi

Department of Pure Mathematics, Faculty of Mathematical Sciences, University of Guilan, Rasht, Iran

E-mail address: aabbasi@guilan.ac.ir

\section{A. Ramin}

Department of Pure Mathematics, Faculty of Mathematical Sciences, University of Guilan, Rasht, Iran

E-mail address: ramin2068@webmail.guilan.ac.ir 